\title{
O papel do cirurgião-dentista com mulheres vítimas de violência doméstica: uma
}

\section{revisão integrativa}

\author{
The role of the dentist with women victims of domestic violence: a integrative review \\ El papel del dentista con las mujeres víctimas de violencia doméstica: una revisón integradora
}

Recebido: 17/01/2022 | Revisado: 26/01/2022 | Aceito: 31/01/2022 | Publicado: 01/02/2022

Luana Garreto Cantanhede

ORCID: https://orcid.org/0000-0002-6946-4490

Centro Universitário Dom Bosco, Brasil

E-mail: garretoluana@gmail.com

Pedro Henrique de Aguiar Moreira

ORCID: https://orcid.org/0000-0002-6228-2467

Universidade CEUMA, Brasil

E-mail: opedrohenrique@yahoo.com

Daniella de Oliveira da Silva

ORCID: https://orcid.org/0000-0002-6325-3027 Universidade CEUMA, Brasil

E-mail: daniella.ortho@gmail.com

Danielli Maria Zucateli Feitosa

ORCID: https://orcid.org/0000-0003-2429-7120

Centro Universitário Dom Bosco, Brasil

E-mail: danielli.odonto@hotmail.com

Andres Felipe Millan Cardenas

ORCID: https://orcid.org/0000-0002-7434-3327 Universidade CEUMA, Brasil

E-mail: andresfelipemillancardenas@hotmail.com

Fabiana Suelen Figuêredo de Siqueira

ORCID: https://orcid.org/0000-0002-1079-3476

Universidade CEUMA, Brasil

E-mail: fabisfsiqueira@hotmail.com

\begin{abstract}
Resumo
O objetivo deste trabalho foi demostrar a importância da correta conduta do cirurgião-dentista no atendimento inicial, deteção e notificação de casos de mulheres vítimas de violência doméstica, bem como seu papel na reabilitação oral. Este trabalho foi classificado como uma revisão integrativa da literatura de pesquisa descritiva e abordagem qualitativa. Os levantamentos bibliográficos foram feitos através de bases de dados online, destacando-se, Pubmed, BBO, ABOL e Scielo. Foram selecionados artigos publicados entre 2010 e 2021 nos idiomas português e inglês. As pesquisas comprovam que as raízes da violência de gênero são profundas, pois advém de uma construção social patriarcal. Após a análise, a revisão de literatura demostrou que os profissionais fazem encaminhamentos inadequados, encaminhando apenas para um serviço/profissional (principalmente Delegacia de Defesa da Mulher ou psicólogo), sem acompanhar o caso, o que é considerado insuficiente em casos de violência contra mulher, além de ir contra o princípio de integralidade. Conclui-se que os profissionais de saúde não possuem uma conduta satisfatória com pacientes vítimas de violência doméstica, tanto na detecção de casos como na notificação, principalmente por despreparo, desconhecimento e desconfiança da resolutividade dos casos pelo serviço público, sendo necessária a implementação de debates sobre o assunto nos ambientes acadêmicos e profissionais.
\end{abstract}

Palavras-chave: Violência doméstica; Violência contra mulher; Odontologia legal.

\begin{abstract}
The aim of this study is demonstrate the importance of correct conduct by dentists in the initial care, detection and notification of cases of women victims of domestic violence, as well as the role in the oral rehabilitation. This work was classified as a descriptive literature review and qualitative approach. The review was made using online databases, Pubmed, BBO, ABOL and Scielo. Articles published between 2010 and 2021, in Portuguese and English were revised. After full-text analyses, the review showed that the professionals make inappropriate referrals, referring only to a service/professional (mainly the Women's Defense Police Station or a psychologist), without following up on the case, which is considered insufficient in cases of violence against women, in addition to going against the principle of integrality. It is concluded that health professionals do not have a satisfactory behavior with patients who are victims of domestic violence, both in case detection and notification, mainly due to lack of preparation, ignorance
\end{abstract}


and distrust of the resolution of cases by the public service, requiring the implementation of debates on the subject in academic and professional environments.

Keywords: Domestic violence; Violence against women; Forensic dentistry.

\section{Resumen}

El objetivo de este estudio fue demostrar la importancia de la correcta conducta de los odontólogos en la atención inicial, detección y notificación de mujeres víctimas de violencia intrafamiliar, así como su papel en la rehabilitación oral. Este trabajo fue cataloga como una revisión bibliográfica de investigación descriptiva y abordaje cualitativo, las encuestas bibliográficas se realizaron a través de bases de datos en línea, destacando, Pubmed, BBO, ABOL y Scielo. Se seleccionaron artículos publicados entre 2010 y 2021 en portugués e inglés. Después de analizar, la revisión de literatura demostró que los profesionales realizan derivaciones inadecuadas, remitiendo únicamente a un servicio/profesional (principalmente Comisaría de Defensa de la Mujer o psicóloga), sin seguimiento del caso, lo que se considera insuficiente en casos de violencia contra la mujer, además de ir en contra del principio de integridad. Se concluye que los profesionales de la salud no tienen un comportamiento satisfactorio con las pacientes víctimas de violencia intrafamiliar, tanto en la detección de casos como en la notificación, principalmente por falta de preparación, desconocimiento y desconfianza en la resolución de casos por parte del servicio público, requiriendo la Implementación de debates sobre el tema en entornos académicos y profesionales.

Palabras clave: Violencia intrafamiliar; La violencia contra las mujeres; Odontología forense.

\section{Introdução}

Atos violentos que acontecem dentro dos lares, a princípio, enquadram-se no conceito de violência intrafamiliar, que pode ser entendida como toda ação que fira a integridade física, psicológica, o bem-estar e a liberdade de um membro da família (mesmo sem laços de sangue). Geralmente essa violência é cometida por alguém que esteja na posição de poder e autoridade, tal barbárie afeta principalmente mulheres, crianças, idosos e deficientes mentais ou físicos. $\mathrm{O}$ termo "violência doméstica" inclui pessoas que convivem no ambiente familiar, mas não necessariamente são da família, como empregados, agregados e também inclui a agressão feita pelo parceiro íntimo (Abdo et al., 2020; Day et al., 2003).

A violência contra mulher é caracterizada como qualquer ato de violência de gênero que possa causar ou cause danos em âmbito físico, sexual ou psicológico para mulher, seja na vida pública ou privada. Essa problemática é tão urgente e preocupante que segundo a Organização das Nações Unidas/ONU Mulheres (ONU, 2017), 40\% das mulheres brasileiras já foram vítimas de violência doméstica em algum período da vida, e ainda, a pesquisa aponta que dentre 83 países, o Brasil ocupa a $5^{\mathrm{a}}$ posição do ranking em casos de feminicídio, em que uma mulher é morta a cada 2 horas em território nacional (da Silva, 2019; ONU, 2017).

Ainda, a violência doméstica contra a mulher quase sempre é acompanhada de violência psicológica e sexo forçado (Day et al., 2003). Esse tipo de violência não se classifica como violência interpessoal, pois nesse caso, homens estão mais sujeitos a serem vítimas de estranhos, enquanto mulheres costumam ser vítimas de membros da própria família e de parceiros íntimos. Dados apontam o quanto esse assunto é urgente: os parceiros íntimos cometem cerca de 40 a $70 \%$ dos homicídios femininos no mundo (Day et al., 2003).

Mesmo com o medo e vivendo sob constante ameaça, algumas mulheres conseguem abandonar os parceiros violentos, principalmente quando as agressões são intensificadas. Mulheres mais jovens são mais propensas a sair desse meio, principalmente quando possuem apoio familiar e quando os filhos são envolvidos na agressão. Porém, somente a separação não é o suficiente, visto que os riscos de assassinato pelo parceiro são maiores após a separação, por isso a importância de leis que protejam as vítimas através da intervenção de políticas públicas (da Silva, 2019; de Lima \& Werlang, 2011).

Sabe-se que quando há violência doméstica do tipo física e/ou sexual, comumente a vítima apresenta traumas na região da face, como ao redor dos olhos, seios da face, lábios, dentição e cavidade oral, logo, o Cirurgião-dentista (CD) tem um papel importante na detecção precoce, acompanhamento e notificação de violência aos órgãos competentes. Adicionalmente, os profissionais de saúde são os primeiros a entrar em contato com as vítimas, que buscam tratar as lesões de face (Costa et al., 2013). 
Vale ressaltar, que é dever dos profissionais de qualquer área da saúde notificar casos de suspeita ou confirmação de maus-tratos, sob pena de multa se não notificarem, de acordo com o Decreto-lei 3.688 de 1941 (Brasil, 1941). Infelizmente, a maioria dos profissionais não notifica, seja por não saber como, não acreditar no poder judiciário ou não ter recebido o conhecimento necessário, ainda na faculdade, para lidar com situações de violência e mazelas sociais, de forma a não ver o paciente como um todo, tratando somente a "queixa principal" e não a etiologia (Carvalho et al., 2013).

Logo, os profissionais de saúde tendem a tratar os ferimentos, mas não costumam abordar a questão com o paciente e nem notificam aos órgãos competentes, através da ficha de notificação que é encaminhada ao serviço social ou ao Programa de Prevenção e Atendimento às Vítimas de Violência - PAV, da Unidade de Saúde. Sabe-se que as alterações estéticas decorrentes de violência física causam distúrbios psicológicos e, nesses casos, a importância da estética vai além de parâmetros físicos, pois atua devolvendo qualidade de vida e autoestima ao paciente (Salazar-Gamarraa et al., 2015).

$\mathrm{Na}$ odontologia, o CD além de ter que notificar a agressão, pode atuar em conjunto com uma equipe multiprofissional, devolvendo a função, estética e autoestima através da reabilitação oral. Todavia, existem poucos casos sobre a atuação do cirurgião-dentista relatados na literatura, levando à suspeita de pouca atuação odontológica na reabilitação e notificação de pacientes em situações de violência doméstica. Essa prática faz com que a violência doméstica não seja vista como um problema de saúde pública e dificulta as políticas de saúde levantadas para combater a violência. Além disso, o pouco acesso às intervenções odontológicas e estéticas prejudica o prognóstico dessas vítimas (Carvalho et al., 2013).

Dessa forma, o presente estudo tem como objetivo relatar o histórico da violência doméstica contra a mulher, enfatizando a natureza e a prevalência das lesões, demonstrando a importância da correta conduta do cirurgião-dentista no atendimento inicial e tratamento de mulheres vítimas de violência doméstica, uma vez que seguir a conduta adequada contribui significativamente para o levantamento de políticas públicas que enfrentem e combatam esse tipo de violência.

\section{Metodologia}

Refere-se a uma revisão integrativa da literatura, de natureza descritiva com abordagem qualitativa, uma vez que o tema escolhido fornecerá resultados através da percepção e análise de dados subjetivos.

A coleta de dados foi feita através de levantamentos bibliográficos realizados nas de bases de dados online Pubmed, web of science, LILACS/Scielo, BBO e Google Acadêmico acessados entre os meses de Junho a Novembro de 2021. Sendo utilizado o operador booleano "AND" para busca simultânea dos descritores. As palavras-chave para essa pesquisa, retiradas do DECS (Descritores em Ciências da Saúde) foram: "Violência Doméstica" (Domestic Violence)"; "Violência contra mulher" (Violence Against Women) e "Odontologia Legal" (Forensic Dentistry). A busca também foi realizada na literatura cinzenta e de forma manual nas referências dos artigos selecionados.

Como critérios de inclusão, foram selecionados artigos publicados entre 2010 e 2021 nos idiomas português e inglês. Revisão, identificação e seleção dos artigos que atendessem aos objetivos da pesquisa, foi realizada por meio de análise dos títulos e resumos, por dois avaliadores cegos, seguindo os seguintes critérios de inclusão: 1- Profissionais da área odontológica de ambos os sexos, independentemente do nível de escolaridade e grau de formação profissional; 2- Violência doméstica contra mulher; 3- Desenho do estudo: Estudos observacionais e clínicos. Quaisquer divergências em quaisquer momentos foram resolvidas por um terceiro pesquisados.

A busca total revelou 376 artigos através dos descritores selecionados nas bases de dados. As evidências duplicadas foram localizadas com ajuda do Mendeley e excluídas, obtendo-se 22 artigos relevantes ao objetivo da pesquisa como fonte de construção à fundamentação teórica para leitura na integra, os demais foram desconsiderados por não atender as propostas da pesquisa. (Figura 1) 
Figura 1. Fluxograma da busca e seleção dos estudos.
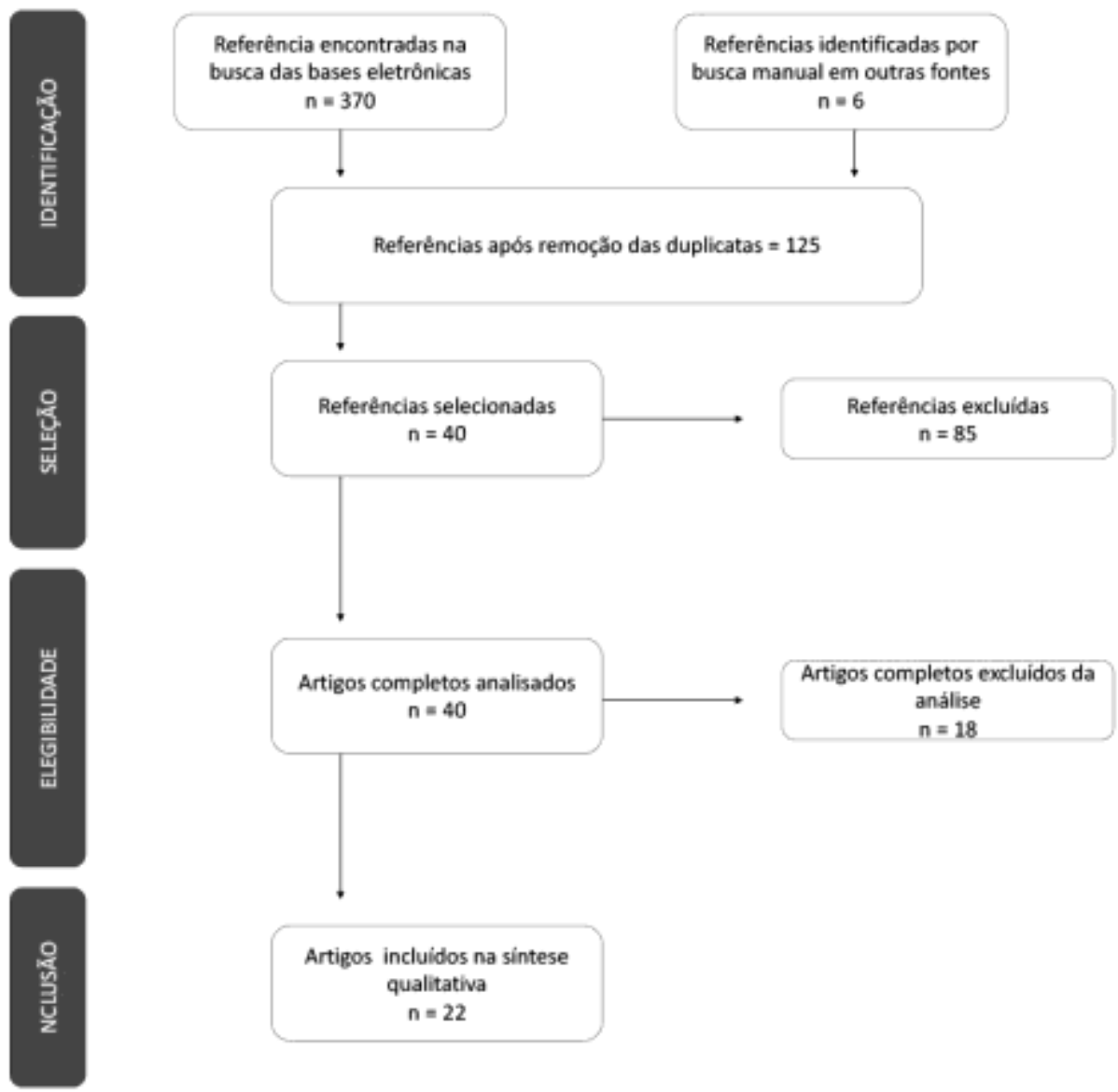

Fonte: Autores.

A sumarização dos artigos selecionados segundo título, autor, ano, objetivo, tipo de estudo, e principais resultados foram realizadas e após esses estudos foram avaliados conforme a escala Jadad Modificada (Jadad et al., 1996) por dois avaliadores independentes com auxílio de um formulário desenvolvido pela equipe da pesquisa e quaisquer divergências foi resolvida por um terceiro avaliador. (Tabela 1)

Tabela 1. Sumarização de artigos selecionados de acordo com o título, autores e principais resultados.

\begin{tabular}{l|c|l}
\hline \multicolumn{1}{c|}{ Titulo } & Autores & \multicolumn{1}{c}{ Principais resultados } \\
\hline $\begin{array}{l}\text { Violência contra a mulher: notificação } \\
\text { compulsória e outros instrumentos legais de uso } \\
\text { dos profissionais de saúde }\end{array}$ & $\begin{array}{c}\text { (Santinon et } \\
\text { al., 2010) }\end{array}$ & $\begin{array}{l}\text { O conhecimento da legislação específica quanto à notificação } \\
\text { compulsória e a inclusão participativa dos profissionais de saúde nos } \\
\text { programas governamentais relativos ao tema não é apenas uma } \\
\text { obrigação legal, mas uma prova de cidadania e valorização do } \\
\text { próximo. }\end{array}$ \\
\hline $\begin{array}{l}\text { A responsabilidade dos profissionais de saúde na } \\
\text { notificação dos casos de violência contra crianças } \\
\text { e adolescentes de acordo com seus códigos de } \\
\text { ética }\end{array}$ & $\begin{array}{c}\text { (de Almeida et } \\
\text { al., 2012) }\end{array}$ & $\begin{array}{l}\text { Cobrigatoriedade da notificaçãa em casos de violência, e que os } \\
\text { profissionais têm o dever de fazê-lo, podendo ser responsabilizados } \\
\text { por omissão ou negligência de acordo com o Estatuto da Criança e } \\
\text { do Adolescente. }\end{array}$ \\
\hline $\begin{array}{l}\text { Educational needs in the field of detection of } \\
\text { domestic violence and neglect: The opinion of a } \\
\text { population of French dentists }\end{array}$ & $\begin{array}{c}\text { (Divakar et al., } \\
2019)\end{array}$ & $\begin{array}{l}\text { Mais informações e treinamento sobre abuso e violência doméstica } \\
\text { são necessários nos cursos de odontologia na França, para ajudar aos } \\
\text { profissionais a lidar com esses pacientes. }\end{array}$ \\
\hline $\begin{array}{l}\text { O cirurgião-dentista frente à violência doméstica: } \\
\text { conhecimento dos profissionais em âmbito } \\
\text { público e privado }\end{array}$ & $\begin{array}{c}\text { (Carvalho et } \\
\text { al., 2013) }\end{array}$ & $\begin{array}{l}\text { Os profissionais analisados conhecem a importância do cirurgião- } \\
\text { dentista no que se refere à violência doméstica, mas apresentam } \\
\text { dificuldades na identificação e nos procedimentos frente à violência. }\end{array}$ \\
\hline
\end{tabular}


Assistência multiprofissional à mulher vítima de violência: atuação de profissionais e dificuldades encontradas.

\section{(Costa et al.,} 2013)

Como os profissionais de saúde atendem mulheres em situação de violência? Uma análise triangulada de dados.
(Hasse \&

Vieira, 2014)

Enfatiza-se a necessidade de maior interação da rede de saúde com os serviços especializados, buscando aprofundar as questões preventivas e educativas relacionadas à mulher e sua família, como forma de oferecer melhor suporte a todos profissionais da área da saúde que lidam com a causa

A violência de gênero deve ser abordada na formação de profissionais de saúde, processo que deve continuar nos serviços, objetivando sensibilizar e preparar esses profissionais para lidar com o tema. Além de desenvolver atividades preventivas e identificar a rede de proteção contra a violência é urgente para o enfrentamento do problema.

A maioria dos profissionais da Estratégia Saúde da Família de um município de São Paulo encontra-se despreparada para o

Reconhecimento e notificação de violência pelos profissionais da estratégia de saúde da família
(Garbin et al., 2016)

Violência doméstica e familiar contra a mulher: estudo de casos e controles com vítimas atendidas em serviços de urgência e emergência.

(Garcia et al., 2016) reconhecimento e notificação dessas ocorrências. Por tanto, o aprimoramento das estratégias, de modo a capacitar estes profissionais para realizar um atendimento adequado às vítimas de violência intrafamiliar deve ser realizado.

A violência doméstica e familiar teve o consumo de bebida alcoólica como fator fortemente associado. Os dias e horas de maior ocorrência evidenciam a necessidade de adequação dos serviços de atendimento às vítimas.

Lesões na cabeça, pescoço e face são muito comuns na vitima de violência domestica, e os Cirurgiões-dentistas têm a oportunidade de

Oral health care providers' knowledge and attitudes about intimate partner violence.

(Harris et al., 2016)

The role and education of dental care professionals in identifying domestic violence: Report of an audience participation exercise and round table discussion.

Facial trauma as physical violence markers against elderly Brazilians: A comparative analysis between genders

Violence against women: caracteristics of head and neck injuries

Violência contra mulher relacionada ao trauma de
face.

(de Castro et al., 2017)

(Malachias, 2017)

Prevalência de traumatismos maxilofaciais causados por agressão ou violência física em mulheres adultas e os fatores associados.
(Chaves et al., 2018)
(Lea et al., 2017)

(de Sousa et al., 2016) desempenhar um papel fundamental no gerenciamento da "epidemia silenciosa" da violência doméstica, incluindo pacientes novos e os que são atendidos rotineiramente.

Apenas o $42 \%$ considerou que a violência domestica é adequadamente relatada pelos cirurgiões-dentistas e que falta clareza sobre a responsabilidade profissional.

Existe uma alta prevalência de lesões faciais entre brasileiros vítimas de violência física e há diferenças importantes relacionadas às características de vitimização de acordo com o sexo na população Brasileira.

À violência contra a mulher são de grande importância e devem ser destacadas e divulgadas, para incentivar a implementação de políticas públicas e ações práticas de acolhimento e reconhecimento dessa violência, incluindo a inserção do Cirurgião-dentista na equipe de saúde.

Os dentistas tem a obrigação de notificar casos de violência doméstica pelo parceiro, e o descumprimento dessa obrigação pode leva-lo à responder legal e eticamente por omissão.

A prevalência de traumatismos maxilofaciais por violência em mulheres é alta, especialmente relacionada à idade, à dependência econômica, ao grau de escolaridade, à ocupação e a problemas psicoafetivos que podem também influenciar a dinâmica familiar.

Os cirurgiões-dentistas que atuam na rede pública dos municípios da região carbonífera demostraram pouco conhecimento quanto aos tipos de violência, a notificação compulsória de violência e ao encaminhamento das vítimas.

(Fernandes et al., 2018)

cirurgiões-dentistas que atuam na Rega Carbonífera, SC.

\begin{abstract}
Intimate partner violence screening in the dental setting: Results of a nationally representative survey.
\end{abstract}

A inclusão de breves intervenções focadas em violência domestica no processo de formação do cirurgião-dentista, bem como o estabelecimento de colaborações entre o dentista e as agências governamentais, em conjunto com uma mudança geral nas atitudes dos dentistas sobre suas responsabilidades profissionais podem facilitar a adoção de triagem de casos de violência domestica no ambiente odontológico.

Análise pericial das lesões situadas em cabeça e pescoço de mulheres vítimas de violência doméstica atendidas em um instituto médico legal de Maceió-AL
(Parish et al., 2018)

(Soares et al., 2018)
Importância de laudos fornecidos pelo odontolegista que auxiliam no esclarecimento de incidências e na identificação dos tipos de lesões da cabeça e pescoço identificados em vítimas de agressão doméstica. 


\begin{tabular}{l|c|l}
\hline $\begin{array}{l}\text { A odontologia e a violência doméstica contra } \\
\text { mulheres: diagnóstico e conduta }\end{array}$ & $\begin{array}{c}\text { O profissional além de diagnosticar e tratar deve utilizar a } \\
\text { 2019) }\end{array}$ & $\begin{array}{l}\text { lotificação compulsória como uma importante ferramenta de } \\
\text { combate e de garantia de direitos. Mas, para isso, é necessária uma } \\
\text { melhor preparação destes, com intuito de formar não só profissionais } \\
\text { melhores, como mais humanos. }\end{array}$ \\
\hline $\begin{array}{l}\text { Análise temporal das agressões físicas contra a } \\
\text { mulher sob a perspectiva da odontologia legal na } \\
\text { cidade de Fortaleza, Ceará }\end{array}$ & $\begin{array}{c}\text { (de Oliveira et } \\
\text { al., 2019) }\end{array}$ & $\begin{array}{l}\text { A Lei Maria da Penha promoveu um impacto positivo na violência } \\
\text { doméstica, ao reduzir a gravidade das lesões localizadas na região } \\
\text { bucomaxilofacial. }\end{array}$ \\
\hline $\begin{array}{l}\text { Trauma bucomaxilofacial resultado da violência } \\
\text { doméstica contra a mulher. }\end{array}$ & $\begin{array}{l}\text { (Pereira et al., } \\
\text { 2019) }\end{array}$ & $\begin{array}{l}\text { O cirurgião dentista está entre os profissionais que mais tem chances } \\
\text { de identificar a violência doméstica, uma vez que a face é a região } \\
\text { preferida dos Agressores para desferir golpes, o que provoca lesões } \\
\text { graves e que exigem Cuidados de profissionais específicos da área. }\end{array}$ \\
\hline $\begin{array}{l}\text { Alarming trends in US domestic violence during } \\
\text { the COVID-19 pandemic. }\end{array}$ & $\begin{array}{l}\text { Com o aparente aumento nos relatórios de violência doméstica, há } \\
\text { necessidade de modalidades mais atuais e padronizadas de relatar } \\
\text { dados de violência doméstica. Os médicos, cirurgiões-dentistas e } \\
\text { outros profissionais de saúde precisam estar cientes do potencial de } \\
\text { aumento da violência intrafamiliar doméstica durante a pandemia de } \\
\text { COVID-19 para que possam responder adequadamente. }\end{array}$ \\
\hline $\begin{array}{l}\text { Violência doméstica: A importância da formação } \\
\text { do Cirurgião-Dentista frente a esse agravo }\end{array}$ & $\begin{array}{l}\text { Há necessidade de uma maior abordagem sobre esse assunto por } \\
\text { parte dos professores que lecionam as disciplinas básicas da grade } \\
\text { curricular, pois, embora os participantes tenham respondido } \\
\text { positivamente às questões, uma insegurança e deficiência em relação } \\
\text { à o tema proposto foi anotado. }\end{array}$ \\
\hline
\end{tabular}

Fonte: Autores.

\section{Resultados e Discussão}

\subsection{Histórico da violência doméstica contra a mulher}

As pesquisas comprovam que as raízes da violência de gênero são profundas, pois advém de uma construção social patriarcal. Sabe-se que os papéis de gênero são passados de geração a geração como se fossem papéis próprios do homem e mulher, entretanto, é válido ressaltar que a construção da violência doméstica contra mulher não está ligada às diferenças biológicas entre os sexos, mas sim aos papéis sociais atribuídos a cada gênero (Bott et al., 2019; Signorelli et al., 2013).

A força masculina sempre dominou a sociedade. $\mathrm{Na}$ antiga sociedade romana, o Estado não punia delitos de mulheres, essa responsabilidade era do homem/marido. Já a Grécia, no século V, considerava somente os homens como cidadãos, as mulheres nascidas em famílias ricas ficavam em casas até a morte, enquanto as mais pobres tinham que trabalhar no mercado e campo (Santiago \& Coelhoi, 2007). Assim, costumes chocantes ocorriam também nas tribos dos primitivos Hindus, em que dois irmãos poderiam ter a mesma esposa e após a morte do marido, era costume incinerar a esposa. No feudalismo, os homens mantinham controle total sobre as esposas, onde, quando viajavam, elas eram obrigadas a usar cintos de castidade, para não duvidarem quanto à legitimidade dos filhos (de Castro et al., 2017; Santiago \& Coelhoi, 2007).

A monogamia surgiu na sociedade para legitimar os filhos dos homens. No Brasil Imperial, casos de adultério passaram a ser punidos pelo código penal em 1830, a esposa adúltera poderia ser presa por até 3 anos e a traição era uma afronta aos direitos do marido (Porto \& Amaral, 2014; Santiago \& Coelhoi, 2007).Entretanto, crimes passionais de homicídio contra a mulher tinham a pena absolvida ou eliminada, de acordo com o artigo 27 do Código Penal Brasileiro de 1890, pois entendia-se que os sentidos e a inteligência do acusado eram anulados pela paixão e emoção súbita, que o levavam a uma insanidade momentânea e a cometer o homicídio, como se fosse um "'crime de paixão" (Santiago \& Coelhoi, 2007).

Ainda, o Código Civil Brasileiro de 1916 decretava que a mulher só poderia trabalhar fora com autorização do marido, uma vez que a inserção da mulher no mercado de trabalho era vista como uma afronta à união da família. Direitos básicos, como direito ao voto feminino no Brasil, só surgiu no ano de 1932 e direito ao ingresso no ensino superior somente em 1879 (Porto \& Amaral, 2014). 
A desigualdade entre homens e mulheres inicia na relação familiar, uma vez que esperam que as mulheres sejam passivas, sensíveis, obedientes, subordinadas e responsáveis pelo lar e pelo cuidado dos filhos e marido; já o homem recebe o papel de chefe e provedor do lar, além de poder em âmbito público. Tal construção valoriza o homem em relação à mulher, pois o primeiro é visto como dominador, enquanto o outro é dominado, dessa forma, a mulher perde autonomia e fica mais suscetível a ser vítima de violência (Gomes et al., 2007)

Entender a relação de gênero é fundamental para analisar toda desigualdade e violência sofrida pelas mulheres em toda história, pois somente quando essa questão foi levantada, ainda no século XX, é que a violência doméstica começou a ser vista como um problema social e de saúde pública. Nos anos 70, as mulheres passam a reivindicar os papéis desqualificados e opressivos que lhes são atribuídos apenas por serem mulheres, gerando seu enclausuramento no lar e sua submissão ao provedor (da Fonseca et al., 2012; Gomes et al., 2007). Mudanças políticas e iniciativas dos movimentos feministas em todo o mundo aos poucos têm possibilitado formas de combate a injustiça e desigualdade de gênero. Em 1993, a violência sexual foi reconhecida como um problema de saúde pública pela Organização Mundial de Saúde. Em 1999, o Ministério de Saúde implementou normas para prevenção e tratamento de sequelas da violência sexual contra mulheres e adolescentes (Brasil, 2006)(Brasil, 2015).

Avanços também foram notórios nos anos 2000. Em 2003, a Secretaria Especial de Políticas para Mulheres da Presidência da República estabeleceu políticas públicas para a melhoria da vida da mulher brasileira. Em 2004, a Política Nacional de Atenção Integral à Saúde da Mulher promoveu melhoria das condições de vida e saúde feminina. A Lei Maria da Penha ( $\mathrm{n}^{\circ}$ 1340/2006) abriu portas para o julgamento de crimes contra a mulher, visando a dignidade humana (Brasil, 2006; Brasil, 2015).

Em 2007, foi criado o Pacto Nacional pelo Enfrentamento da Violência contra a Mulher, que cobra a prevenção e combate à violência, além de garantir os direitos das mulheres nas esferas sociais, familiares, educacionais, do trabalho, da saúde e da segurança pública. Em 2013, foi sancionada a Lei que prevê atendimento integral e multidisciplinar às vítimas de violência sexual em todos os serviços de urgência e emergência do SUS (Brasil, 2013). A partir dessa discussão de gênero levantada, a violência doméstica foi entendida como um fenômeno multifacetado que necessita de intervenção de políticas públicas, destacando-se a implantação de serviço de atenção à mulher vítima de violência, como Delegacia de Proteção às Mulheres (DPMs) e centros de referência de atenção à mulher vítima de violência (Gomes et al., 2007).

\subsection{Consequências físicas e psicológicas à vítima de violência doméstica}

A agressão pelo parceiro íntimo é o tipo de violência mais comum e possui um padrão rotineiro, em que o agressor tem o controle e domina a situação. No dia a dia, esses abusos podem se manifestar através de agressões físicas com golpes, tapas, socos, chutes, surras, estrangulamento, queimaduras, uso de objetos perfurocortantes, quebra de objetos e ameaças de ferir membros próximos. A agressão psicológica ocorre por meio de intimidações, humilhações e menosprezo, coerção sexual e comportamentos de extremo controle, restringindo o acesso da vítima a família e amigos (Boserup et al., 2020; Day et al., 2003; Signorelli et al., 2013).

Segundo (Day et al., 2003), os fatores causais estão relacionados: (1) ao agressor: ser homem, ter sofrido abuso ou presenciado violência na infância, pai ausente, alcoolismo e uso de drogas; (2) à relação: conflito conjugal e controle das finanças pelo parceiro; (3) aos fatores da comunidade: pobreza, desemprego, isolamento das vítimas e família, alta taxa de criminalidade no meio, (4) aos fatores sociais: normatização da violência como método de resolução de conflitos, normas socioculturais que dão ao homem maior poder e domínio, mas desqualificam a mulher, conceito de masculinidade ligado a dominação, honra e agressão. 
Nessa mesma direção, (de Oliveira et al., 2019), fizeram uma pesquisa acerca da ocorrência de lesões na região de cabeça e pescoço de mulheres submetidas ao exame pericial em Maceió/AL e Fortaleza/CE, respectivamente. Todos os autores concordaram quanto aos dados encontrados, chegando à conclusão que a faixa etária de maior prevalência foi entre 30-59 anos, com escolaridade até o ensino médio e ocupação de atividade manual ou donas de casa. Os agressores são companheiros ou excompanheiros, do sexo masculino e o tecido mais afligido foi tecido mole, deixando lesões do tipo equimose, escoriação e edema, com instrumento contundente; ainda, (da Silva et al., 2021) destacaram que a maioria das vítimas foi classificada economicamente como classe D.

Muitas vezes, a mulher é julgada quando continua submetendo-se ao parceiro violento, entretanto, essa atitude está diretamente ligada às limitações que ela possui, como medo de represália, perda de suporte financeiro, falta de suporte familiar, dependência emocional ou preocupação com os filhos. Outros fatores que contribuem para a continuação da relação são: sintomas depressivos; repetição de modelo parental violento; maus-tratos, abandono e violência sexual na infância; sentimento de culpa pelo comportamento violento do parceiro, entre outros (Day et al., 2003).

Os traumas maxilo-faciais provocam graves repercussões estéticas, psicológicas e sociais. Além da região de cabeça e pescoço ser mais exposta e menos "protegida", lesões na face estão diretamente ligadas a humilhação, sentimento comum nas vítimas. As cicatrizes, disfunções mastigatórias e estéticas são lembretes dolorosos do abuso. Além disso, funções do sistema estomatognático como deglutição, fala e mastigação também podem ser afetadas por traumas faciais (Chaves et al., 2018).

Geralmente, as vítimas apresentam certas reações após o trauma, como memórias vividas dos eventos de violência, pesadelos, vigilância constante, ansiedade, medo, abuso de substâncias, problemas sexuais, tristeza, raiva, agressividade, desespero, falta de confiança, auto isolamento. A violência doméstica está mais relacionada ao trauma tipo II, com reações que perduram por mais tempo (diferentemente do trauma tipo I, em que as reações duram menos tempo). A vítima passa a ter sintomas como somatização, mudanças de identidade e percepção do agressor, mudanças no sentido da vida e nas suas relações interpessoais, ansiedade, depressão e estresse pós-traumático são a constatação mais comum nos estudos clínicos das vítimas de violência (Gomes, 2012).

Como a face costuma ser o alvo mais atingido, além de danos físicos, as lesões provocam danos psicológicos, pois ao agredir a face, o agressor atinge diretamente a autoimagem da vítima, o rosto representa uma região privilegiada em todas as culturas e ele carrega as emoções e identidade do indivíduo. Por isso, é comum que as vítimas sintam vergonha, humilhação e culpa. Muitas mulheres param de se maquiar, pois sentem que ao revelar o rosto, também estão expondo a violência sofrida (Soares et al., 2018).

\subsection{Natureza e prevalência das lesões}

A Organização Pan Americana de Saúde dividiu o ciclo de violência contra a mulher em três fases. A primeira é caracterizada pela acumulação da tensão, quando as agressões são "mais leves" e menos frequentes, por isso a vítima costuma ser compreensiva e tenta encontrar um motivo para agressividade, acreditando que não ocorrerá novamente (de Sousa et al., 2016; Soares et al., 2018).

Na segunda fase ocorre a explosão da violência, com agressões mais graves e mais frequentes. A vítima costuma estar à mercê do agressor e já não tem muito controle sobre a situação. Já na terceira fase, a violência e tensão desaparecem por um tempo, o agressor demonstra arrependimento e amabilidade, promete que não acontecerá novamente, enquanto a vítima sente alívio e acredita que é responsabilidade dela manter a paz no lar (de Castro et al., 2017).

O Decreto-Lei 2.848 (07 de dez de 1940) do código penal, no artigo 129, classificou as lesões em leves, graves, gravíssimas e lesões corporais que resultam em morte. Lesões leves causam danos superficiais, como danos à pele, hipoderme, 
vasos arteriais e venosos. Lesões graves geram sequelas temporárias por mais de 30 dias, causando fragilidade de membro, sentido ou função, aceleração do parto e perigo de vida (Brasil, 1940).

Ainda de acordo com o código, lesões gravíssimas são aquelas que resultam em incapacidade permanente de trabalho, enfermidade incurável, perda de membro, sentido ou função, aborto e deformidade permanente. Casos de lesão seguida de morte só podem ser classificados assim se o autor do crime não quis a morte, nem assumiu o risco de matar. Se esses crimes ocorrem no contexto de violência doméstica, a pena para lesões leves é aumentada de 3 meses para 03 anos e o resto é aumentada em 1/3 (Brasil, 1940).

Em relação a prevalência de lesões, um estudo de casos e controles com vítimas atendidas em serviços de urgência e emergência foi realizado usando dados do Sistema de Vigilância de Violências e Acidentes (VIVA), 623 mulheres vítimas de violência doméstica foram selecionadas. Destas, $97,4 \%$ sofreram violência física: $60,7 \%$ com força corporal/espancamento; $20,9 \%$ com objetos perfurocortantes e $11,9 \%$ com objetos contundentes. As agressões resultaram em corte/laceração (46,4\%) e contusão (20,2\%), chamou atenção que a região da cabeça foi a mais atingida (39,5\%)(Garcia et al., 2016).

Em concordância com outros estudos, nota-se uma alta incidência de lesões por instrumentos contundentes, resultando principalmente em equimoses $(28,85 \%)$ e escoriações $(24,59 \%)$. Lesões extraorais foram mais comuns na região orbital $(17,14 \%)$, cervical $(16,63 \%)$ e frontal $(12,51 \%)$. Já em região intraoral, as lesões são mais frequentes em lábios, entretanto, a porcentagem de 11,63\% de lesões dentárias também chamou atenção, pois 50\% dessas lesões causaram fratura dental, principalmente no incisivo superior (41,67\%). Destaca-se que o CD tem papel fundamental na reabilitação oral dessas vítimas, pois os dentes anteriores, principalmente os incisivos superiores, chamam muita atenção no sorriso e afetam absurdamente a autoestima dessas vítimas (de Castro et al., 2017).

Em contrapartida, (Rezende et al., 2007) classificaram a maioria das lesões como "nuas", sem uso de instrumento, que são lesões causadas por tapas, chutes, socos, empurrões e espancamentos. O punho é o mais usado nos ataques, causando fraturas faciais em 30\% das vítimas. Em síntese, o CD pode estar envolvido em casos de violência de duas formas: identificando os sinais de violência ou tratando das lesões em região de cabeça e pescoço, principalmente as intraorais. Portanto, saber a melhor conduta é essencial para lidar com esses pacientes.

\subsection{Conduta e papel do cirurgião-dentista frente as vítimas de violência doméstica}

Sabe-se que o CD está entre os profissionais da saúde que tem a maior possibilidade de entrar em contato com o paciente que sofre violência, doméstica, pois os sinais e lesões encontram-se na região de cabeça e pescoço. Entretanto, muitos não se sentem aptos para diagnosticar, fazer a notificação compulsória e, para piorar, é comum que haja julgamento do profissional por acreditar em crenças ultrapassadas, resultando em uma relação abalada com a paciente, de maneira que o profissional não consiga atuar de forma efetiva (Carvalho et al., 2013; Harris et al., 2016)(Lea et al., 2017; Parish et al., 2018).

(Garbin et al., 2016) encontravam dificuldades para reconhecer e notificar casos de violência, obtiveram como resultados: 75,4\% dos profissionais não conheciam a ficha de notificação de violência e $60 \%$ não se sentiam responsáveis pela notificação. Os dados lamentáveis ressaltam a falta de conhecimento e preparo dos profissionais em lidar com a violência doméstica, colaborando com o elevado índice de vítimas que sofrem diariamente as consequências dessa violência sem suporte adequado.

Ainda, uma pesquisa para identificar o conhecimento e atuação dos cirurgiões-dentistas e estudantes de odontologia frente a violência doméstica, contou com 223 participantes que responderam um questionário sobre o tema. Destes, 70,4\% não sabiam a diferença entre notificação compulsória e denuncia, 32,3\% não se sentiam confortáveis para fazer a notificação compulsória por motivos de medo de represália $(33,3 \%)$, medo $(10,4 \%)$ e não ter conhecimento $(20,8 \%), 48,4 \%$ não saberiam 
identificar lesões suspeitas de violência e 99,1\% acreditam que esse assunto deveria ser mais discutido na graduação (da Luz et al., 2021).

A Portaria No 2.406 de 5 de novembro de 2004 instituiu o Serviço de Notificação Compulsória e a partir disso, a ficha de notificação de violência interpessoal e/ou autoprovocada deve ser preenchida pelo profissional de saúde em casos suspeitos ou confirmados de violência contra a mulher. O profissional preenche duas vias, uma fica no prontuário do paciente e a outra é encaminhada para Vigilância Epidemiológica do município (Fernandes et al., 2018; Santinon et al., 2010).

Muitos profissionais temem notificar e sofrer represálias, entretanto, a notificação compulsória é de origem formal, institucional e sigilosa, isso significa que a notificação não é uma denúncia e não expõe o profissional a possíveis constrangimentos. Vale destacar que a Lei 13.931/19, do dia 10 de dezembro de 2019, obriga os serviços públicos e privados de saúde a comunicar às autoridades policiais suspeitas e confirmação de violência em um prazo de até 24 horas (Da Silva et al., 2021).

Sabe-se que o Código de Ética Odontológico possui alguns incisos que podem ser relacionados a casos de violência doméstica: no artigo 9, incisos VII a IX citam que o profissional deve zelar pela saúde e dignidade do paciente, promover saúde coletiva ao desempenhar suas funções e resguardar o sigilo profissional (que pode ser quebrado para colaboração da justiça em casos previstos em lei, como na violência doméstica); o artigo 11 enquadra como infração ética permitir que o paciente seja desrespeitado, ou seja, do ponto de vista ético a notificação significa cumprir com os deveres do cirurgiãodentista, que deve zelar pela saúde e dignidade do paciente (de Almeida et al., 2012; de Oliveira et al., 2019).

(Hasse \& Vieira, 2014) fizeram uma pesquisa sobre como os profissionais de saúde atendem mulheres em situações de violência, que escancarou ainda mais os embates sofridos na saúde. Foram entrevistados 221 profissionais, em que somente 61 tiveram algum tipo de capacitação para atender essas vítimas; $49 \%$ desses profissionais realizam consulta e encaminhamento, 39,6\% realizam apenas consulta e 11,4\% afirmaram não fazer nada, pois a vítima pediu sigilo (conduta inadequada).

Muitos profissionais fazem encaminhamentos inadequados, encaminhando apenas para um serviço/profissional (principalmente Delegacia de Defesa da Mulher ou psicólogo), sem acompanhar o caso, o que é considerado insuficiente em casos de violência contra mulher, além de ir contra o princípio de integralidade. Outros problemas podem ser elencados, como a demora no atendimento dessas mulheres no setor público, a falta de tato no atendimento, erros quanto às condutas dos profissionais e a chamada "rota crítica" em que a paciente vai para vários serviços várias vezes, gerando desgaste e desistência da procura por ajuda (Saliba et al., 2007).

O atendimento de vítimas de violência doméstica exige o cumprimento de deveres legais e éticos do profissional, geralmente relacionados a notificação compulsória. O profissional deve notificar de forma sigilosa, usando códigos da CID-10 (T74, etc.) na ficha de notificação disponível pelo Sistema de informação de Agravos de Notificação (SINAN,) que é encaminhada às autoridades competentes. Essa notificação é essencial, pois torna possível dimensionar a violência e suas consequências em um nível coletivo/nacional, esses dados facilitam a implantação de políticas públicas de combate à violência doméstica (Drigeard et al., 2012; Hasse \& Vieira, 2014).

Logo, é necessário que o CD não trate somente as sequelas resultantes da agressão, mas atue de forma humanizada e integral, pois o bom acolhimento nesse momento é fundamental para que ocorra vínculo entre vítima e o serviço de saúde. É dever legal do $\mathrm{CD}$ reconhecer as leis e sua correta conduta frente à violência, ainda, quando se trata de violência doméstica contra mulher, é mandatório, segundo a Lei 10.778, que haja a notificação compulsória aos serviços especializados, para ser encaminhada aos órgãos de Vigilância Sanitária (da Silva, 2019). 


\section{Conclusão}

Por meio deste estudo consta-se:

- A importância da presença do CD no atendimento de mulheres vítimas de violência doméstica, uma vez que ele é um dos primeiros profissionais a ter contato com a vítima, ainda na atenção primária de saúde.

- $\quad \mathrm{O}$ CD, ainda possui condutas inadequadas com esses pacientes, destacando-se a falta de conhecimento, o medo de represálias ao notificar, o preconceito com as vítimas, o tratamento curativo e encaminhamentos inadequados, que fazem a vítima andar em círculos.

- Ainda, se faz necessário que as faculdades de odontologia preparem mais os seus alunos para identificação desses casos, bem como na tomada de condutas corretas.

- Embora a literatura seja conclusiva na necessidade de formular melhores condutas e inclusão de treinamentos aos cirurgiões-dentistas para melhorar o atendimento inicial e tratamento de mulheres vítimas de violência doméstica, futuros estudos multicêntricos que avaliem o efeito cultural e regional sobre a violência doméstica ainda precisam ser conduzidos.

\section{Referências}

Abdo, C., Miranda, E. P., Santos, C. S., Junior, J. B., \& Bernardo, W. M. (2020). Domestic violence and substance abuse during COVID19: A systematic review. Indian J Psychiatry, 62(Suppl 3), S337-S342. https://doi.org/10.4103/psychiatry.IndianJPsychiatry_1049_20

Boserup, B., McKenney, M., \& Elkbuli, A. (2020). Alarming trends in US domestic violence during the COVID-19 pandemic. Am J Emerg Med, $38(12)$, 2753-2755. https://doi.org/10.1016/j.ajem.2020.04.077

Bott, S., Guedes, A., Ruiz-Celis, A. P., \& Mendoza, J. A. (2019). Intimate partner violence in the Americas: a systematic review and reanalysis of national prevalence estimates. Rev Panam Salud Publica, 43, e26. https://doi.org/10.26633/RPSP.2019.26

Brasil, Presidência da República (1940). DECRETO-LEI No 2.848, DE $7 \quad$ DE DEZEMBRO DE 1940. Retrieved 30/01/2022 from http://www.planalto.gov.br/ccivil_03/decreto-lei/del2848compilado.htm

Brasil, Presidência da República (1941). DECRETO-LEI No 2.848, DE 7 DE DEZEMBRO DE 1941. 1940. Retrieved 30/01/2022 from http://www.planalto.gov.br/ccivil_03/decreto-lei/del2848compilado.htm

Brasil, Presidência da República (2006). LEI $N^{o} \quad 11.340, \quad D E \quad 7 \quad D E \quad A G O S T O \quad D E \quad 2006 . \quad$ Retrieved $30 / 01 / 2022 \quad$ from http://www.planalto.gov.br/ccivil_03/_ato2004-2006/2006/lei/111340.htm

Brasil, Presidência da República (2013). LEI $N^{o} \quad 12.845, \quad D E \quad 1^{o} \quad$ DE AGOSTO DE 2013 . Retrieved $30 / 01 / 2022 \quad$ from http://www.planalto.gov.br/ccivil_03/_ato2011-2014/2013/lei/112845.htm

Brasil, S. d. P. p. a. m. (2015). Atenção humanizada às pessoas em Situação de violência sexual com Registro de informações e coleta de vestígios. Retrieved 30/01/2022 from https://bvsms.saude.gov.br/bvs/publicacoes/atencao_humanizada_pessoas_violencia_sexual_norma_tecnica.pdf

Carvalho, L. M. F., Galo, R., \& da Silva, R. H. A. (2013). O cirurgião-dentista frente à violência doméstica: conhecimento dos profissionais em âmbito público e privado. Medicina (Riveirão Preto), 46(3), 297 - 304. https://doi.org/https://doi.org/10.11606/issn.2176-7262.v46i3p297-304

Chaves, A. d. S., Lund, R. G., Martos, J., Salas, M. M. S., \& Soares, M. R. P. S. (2018). Prevalência de traumatismos maxilofaciais causados por agressão ou violência física em mulheres adultas e os fatores associados: uma revisão de literatura. Revista da faculdade de odontologia da universidade de passo fundo (RFO), 23(1), 60 - 67. https://doi.org/ https://doi.org/10.5335/rfo.v23i1.8081

Costa, D. A. C., Marques, J. F., Moreira, K. d. A. P., Gomes, L. F. d. S., Henriques, A. C. P. T., \& Fernandes, A. F. C. (2013). Assistência multiprofissional à mulher vítima de violência: Atuação de profissionais e dificuldades encontradas. Revista Cogitare Enfermagem, 18(2), 301 - 309. https://doi.org/http://dx.doi.org/10.5380/ce.v18i2.29524

da Fonseca, D. H., Ribeiro, C. G., \& Leal, N. S. B. (2012). Violência doméstica contra a mulher: realidades e representações sociais. Psicologia \& Sociedade, 24(2), 307 - 314. https://doi.org/https://doi.org/10.1590/S0102-71822012000200008.

da Luz, M. F. S., Loreto, D. B. L., \& de Barros, B. A. C. (2021). Conhecimento e atuação de cirurgiões-dentistas e estudantes de odontologia frente à violência contra a mulher, com ênfase na notificação compulsória. Revista Brasileira de Odontologia Legal (RBOL), 8(2), 26 - 35.

da Silva, A. O., Silva, M. d. C. L. C., de Godoy, A. B., da Silva, L. M. R. C., \& Soares, A. L. F. d. H. (2021). Domestic violence: The importance of the training of the Dental Surgeon against this condition. Research, Society and Development, 10(5), 1 - 9. https://doi.org/http://dx.doi.org/10.33448/rsdv10i5.14654

da Silva, E. D. M. (2019). A odontologia e a violência doméstica contra mulheres: diagnóstico e conduta. Scire Salutis Journal, 9(3), 22 - 32. https://doi.org/https://doi.org/10.6008/CBPC2236-9600.2019.003.0004 
Day, V. P., Telles, L. E. d. B., Zoratto, P. H., de Azambuja, M. R. F., Machado, D. A., Silveira, M. B., \& Blank, P. (2003). Violência doméstica e suas diferentes manifestações. Revista de Psiquiatria do Rio Grande do Sul 25, 9-21. https://doi.org/https://doi.org/10.1590/S0101-81082003000400003

de Almeida, A. H. d. V., da Silva, M. L. C. A., Musse, J. d. O., \& Marques, J. A. M. (2012). A responsabilidade dos profissionais de saúde na notificação dos casos de violência contra crianças e adolescentes de acordo com seus códigos de ética. Arquivos em Odontologia, $48(2)$, 109 - 115. https://doi.org/10.7308/aodontol/2012.48.2.08

de Castro, T. L., Tinoco, R. L. R., Lima, L. N. C., Costa, L. R. d. S., Francesquini Júnior, L., \& Daruge Júnior, E. (2017). Violence against women: caracteristics of head and neck injuries Revista Gaúcha de Odontologia, 65(2), 100 - 108. https://doi.org/https://doi.org/10.1590/1981863720170002000013245

de Lima, G. Q., \& Werlang, B. S. G. (2011). Women who suffer domestic violence: contributions of psychoanalysis. Psicologia em Estudo, $14(4)$, 511 - 520.

de Oliveira, M. V. J., Lima, M. R. P., Silveira, G. M., Correira, A. d. M., de Almeida, M. E. L., \& Teixeira, A. K. M. (2019). Análise temporal das agressões físicas contra a mulher sob a perspectiva da odontologia legal na cidade de Fortaleza, Ceará. Revista Brasileira de Odontologia Legal (RBOL), 6(3), 02 - 14. https://doi.org/https://doi.org/10.21117/rbol.v6i3.251

de Sousa, R. I., de Macedo Bernardino, I., Castro, R. D., Cavalcanti, A. L., Bento, P. M., \& d'Avila, S. (2016). Facial trauma as physical violence markers against elderly Brazilians: A comparative analysis between genders. Arch Gerontol Geriatr, 67, 55-60. https://doi.org/10.1016/j.archger.2016.06.015

Divakar, U., Nazeha, N., Posadzki, P., Jarbrink, K., Bajpai, R., Ho, A. H. Y., . . Car, J. (2019). Digital Education of Health Professionals on the Management of Domestic Violence: Systematic Review and Meta-Analysis by the Digital Health Education Collaboration. J Med Internet Res, 21(5), e13868. https://doi.org/10.2196/13868

Drigeard, C., Nicolas, E., Hansjacob, A., \& Roger-Leroi, V. (2012). Educational needs in the field of detection of domestic violence and neglect: the opinion of a population of French dentists. Eur J Dent Educ, 16(3), 156-165. https://doi.org/10.1111/j.1600-0579.2012.00739.x

Fernandes, T. B., Rocha, M. P., Losso, A. R. d. S., \& Sonego, F. G. F. (2018). Notificação de violência: conhecimento de cirurgiões-dentistas que atuam na Região Carbonífera, SC. Revista da ABENO, 18(2), 124 - 134. https://doi.org/https://doi.org/10.30979/rev.abeno.v18i2.482

Garbin, C. A. S., Rovida, T. A. S., Costa, A. A., \& Garbin, A. J. I. (2016). Reconhecimento e notificação de violência pelos profissionais da estratégia de saúde da família. Archives of Health Investigation, 5(1), 8 - 12. https://doi.org/https://doi.org/10.21270/archi.v5i1.1294

Garcia, L. P., Duarte, E. C., de Freitas, L. R. S., \& da Silva, G. D. M. (2016). Violência doméstica e familiar contra a mulher: estudo de casos e controles com vítimas atendidas em serviços de urgência e emergência. Cadernos de Saúde Pública, 32(4), 1 - 11. https://doi.org/doi.org/10.1590/0102-311X00011415

Gomes, N. P., Diniz, N. M. F., Araújo, A. J. d. S., \& Coelho, T. M. d. F. (2007). Compreendendo a violência doméstica a partir das categorias gênero e geração. Acta Paulista de Enfermagem 20(4), 504 - 508. https://doi.org/https://doi.org/10.1590/S0103-21002007000400020.

Gomes, R. M. (2012). Women victims of domestic violence and posttraumatic stress disorder: a cognitive behavioral approach. Revista de Psiquiatria da IMED, 4(2), 672 - 680. https://doi.org/https://doi.org/10.18256/2175-5027/psico-imed.v4n2p672-680.

Harris, C. M., Boyd, L., Rainchuso, L., \& Rothman, A. T. (2016). Oral Health Care Providers' Knowledge and Attitudes About Intimate Partner Violence. J Dent Hyg, 90(5), 283-296. https://www.ncbi.nlm.nih.gov/pubmed/29118181

Hasse, M., \& Vieira, E. M. (2014). How health professional assist women experiencing violence? A triangulated data analysis. Saúde em Debate, $38(102)$, 482 - 493. https://doi.org/doi.org/10.5935/0103-1104.20140045.

Jadad, A. R., Moore, R. A., Carroll, D., Jenkinson, C., Reynolds, D. J., Gavaghan, D. J., \& McQuay, H. J. (1996). Assessing the quality of reports of randomized clinical trials: is blinding necessary? Control Clin Trials, 17(1), 1-12. https://doi.org/10.1016/0197-2456(95)00134-4

Lea, S. J., Quinn, B., \& Reynolds, P. A. (2017). The Role and Education of Dental Care Professionals in Identifying Domestic Violence: Report of an Audience Participation Exercise and Round Table Discussion. Technology, Knowledge and Learning, 22(2), 219 - 226. https://doi.org/10.1007/s10758-0169293-9

Malachias, R. C. (2017). Violência contra mulher relacionada ao trauma de face Universidade Federal de Minas Gerais]. http://hdl.handle.net/1843/ODONAZWLBG

ONU, Oorganização das Nações Unidas (2017). ONU alerta para os custos da violência contra as mulheres no mundo. Retrieved 30/01/2022 from http://www.onumulheres.org.br/noticias/onu-alerta-para-os-custos-da-violencia-contra-as-mulheres-no-mundo/

Parish, C. L., Pereyra, M. R., Abel, S. N., Siegel, K., Pollack, H. A., \& Metsch, L. R. (2018). Intimate partner violence screening in the dental setting: Results of a nationally representative survey. J Am Dent Assoc, 149(2), 112-121. https://doi.org/10.1016/j.adaj.2017.09.003

Pereira, J. B., Rodrigues, D. C., Blois, M. C., \& de Souza, F. A. (2019). Trauma bucomaxilofacial resultado da violência doméstica contra mulher. Uningá Journal, 56(S3), 169 - 179. https://doi.org/http://revista.uninga.br/index.php/uninga/article/view/942

Porto, M. L., \& Amaral, W. N. d. (2014). Sexual violence against women: history and management. Femina, $42(4), 209$ - 215.

Rezende, E. J. C., Araújo, T. M., Moraes, M. A. S., Santana, J. S. d. S., \& Radiccchi, R. (2007). Lesões buco-dentais em mulheres em situação de violência: um estudo piloto de casos periciados no IML de Belo Horizonte, MG. Revista Brasileira de Epidemiologia, 10(2), 202 - 214. https://doi.org/https://doi.org/10.1590/S1415-790X2007000200008.

Salazar-Gamarraa, R., de Oliveira, J. A. P., \& Dibc, L. L. (2015). A estética em reabilitação bucomaxilofacial. Revista APCD de Estética, $3(1)$, 42 - 52. https://doi.org/10.13140/RG.2.1.4872.8729 
Research, Society and Development, v. 11, n. 2, e46511225837, 2022

(CC BY 4.0) | ISSN 2525-3409 | DOI: http://dx.doi.org/10.33448/rsd-v11i2.25837

Saliba, O., Garbin, C. A. S., Garbin, A. J. I., \& Dossi, A. P. (2007). Responsabilidade do profissional de saúde sobre a notificação de casos de violência doméstica. Revista de Saúde Pública 41(3), 472 - 477. https://doi.org/https://doi.org/10.1590/S0034-89102007000300021.

Santiago, R. A., \& Coelhoi, M. T. Á. D. (2007). A violência contra a mulher: Antecedentes históricos. revista Unifacs, $11(1), 1$ - 19.

Santinon, E. P., Rosa, G. D. M., \& da Silva, L. C. F. P. (2010). Violência contra a mulher: notificação compulsória e outros instrumentos legais de uso dos profissionais de saúde. Âmbito Jurídico, XIII(4), $1 \quad$ - $\quad 4 . \quad$ https://doi.org/http://www.ambitojuridico.com.br/site/?n_link=revista_artigos_leitura\&artigo_id=7499\&revista_caderno=4

Signorelli, M. C., Auad, D., \& Pereira, P. P. G. (2013). Violência doméstica contra mulheres e a atuação profissional na atenção primária à saúde: um estudo etnográfico em Matinhos, Paraná, Brasil. Cadernos de Saúde Pública [online], 29(6), 1230 - 1240. https://doi.org/https://doi.org/10.1590/S0102311 X2013000600019.

Soares, É. M. G., Cavalcanti, R. R., Wanderley, A. E. C., Souto, R. R. F. A., Lessa, R. M., \& Tenório Neto, J. F. (2018). Análise pericial das lesões situadas em cabeça e pescoço de mulheres vítimas de violência doméstica atendidas em um instituto médico legal de Maceió - AL. Rev. Bras. Odontol. Leg. RBOL, 5(3), 12 - 22. https://doi.org/https://doi.org/10.21117/rbol.v5i3.186 